\title{
AC 2007-372: VHDL PROJECTS TO REINFORCE COMPUTER ARCHITECTURE CLASSROOM INSTRUCTION
}

\section{Ronald Hayne, The Citadel}

Ronald J. Hayne, $\mathrm{PhD}$, is an Assistant Professor in the Department of Electrical and Computer Engineering at The Citadel. His professional areas of interest are digital systems and hardware description languages. He is a retired Army officer with experience in academics and Defense laboratories. 


\title{
VHDL Projects to Reinforce Computer Architecture Classroom Instruction
}

\begin{abstract}
Exploration of various computer architecture constructs needs reinforcement beyond pencil and paper homework problems. Unfortunately, laboratory exercises based on microprocessor trainers are limited to a single architecture and a resolution of single assembly language instructions. A hardware description language, such as VHDL, can be used to provide simulation-based application of the classroom instruction regardless of the course text. Models of computer components such as registers, memory, and ALUs can be readily defined to match textbook examples and then combined to demonstrate multiple architectural concepts. Students with basic knowledge of VHDL from their prerequisite digital logic course are able to modify and use these models to simulate computer behavior at the register transfer level with data and control signal visibility at each clock cycle.
\end{abstract}

A program of instruction has been developed that uses VHDL homework exercises and a capstone design project to provide hands-on application of course concepts using modern design tools. Exercises include addressing modes, microprogrammed control, and computer arithmetic. The design project models a multi-bus architecture and hardwired control unit from the text to implement a basic instruction set. An example assembly language program can be loaded into memory and executed in simulation on the model computer. Results are verified by monitoring control signals, buses, and registers, as well as final dumps of memory and register contents.

Student feedback has been very positive that the VHDL exercises provided reinforcement of classroom concepts and allowed them to visualize results via simulation. Over two-thirds of the students were able to implement a completely functional design project computer model which successfully executed the test program. The methodology was to combine/modify instructor provided VHDL models, rather than turning the course into a "programming” class. This kept the focus on "hardware description" and did not rely on coverage of the language by the course text. The resulting combination of textbook and classroom instruction with VHDL modeling and simulation exercises provided students with a more robust learning experience and exposure to state-of-the-art design tools.

\section{Introduction}

Teaching computer architecture effectively requires reinforcement beyond pencil and paper homework problems. Some form of hands-on laboratory exercises are desirable to provide practical observation of the classroom constructs. Microprocessor trainers are one option, but unless investment is made in multiple systems, exercises are limited to a single architecture. With a resolution of single assembly language instructions, the focus often shifts to assembly language programming of the target architecture, rather than allowing exploration of various computer architecture concepts. 
Hardware description languages such as VHDL provide another alternative for laboratory exercises to supplement classroom instruction. Models can be developed for multiple computer architectures and their function can be observed through simulation. VHDL modeling techniques range from structural interconnection of hardware components to abstract behavioral models with little relation to the underlying hardware. Many texts have been written to teach VHDL, but their focus is most often on the language and its application to large-scale digital systems.

The approach taken here was to develop a set of VHDL exercises to supplement an existing course for teaching computer architecture. The current course text, Computer Organization ${ }^{1}$, contains descriptions of hardware for multiple computer architectures, but no coverage of VHDL or other models of that hardware. A program of instruction has been developed which augments the classroom instruction with VHDL homework exercises and a capstone design project. The focus is kept on demonstration of architectural concepts, rather than turning into a VHDL "programming” course.

\section{Course Content}

The existing course and text cover a wide range of computer architecture constructs which can be supplemented with homework exercises. A fundamental concept is the discussion of various addressing modes used to specify the location of operands in an assembly language program. These addressing modes are first discussed generically and then specifically for several modern computer architectures. Visualization of the addresses and operands is often difficult for students, so an exercise was developed to provide a VHDL simulation to demonstrate the various results.

Another important concept is that of microprogrammed control of the operation of a simple processor. Several control schemes are discussed along with the basis for forming control words. Another homework exercise has been developed to allow the students hands-on application of these concepts. The computer architecture from the addressing mode exercise is coupled with a VHDL model of a microprogram controller which generates the control signals from student coded microinstructions.

Additional exercises are provided to demonstrate control signals and data flow for various processor architectures. Of note is the discussion of computer arithmetic and the implementation of two's complement multiplication via the Booth algorithm, which will not be covered in this paper. Hardware models from the text are implemented in VHDL, again allowing the students simulation-based application of the course concepts.

Many of the course concepts are tied together in a capstone design project which implements a simple processor using a multi-bus architecture and a hardwired control unit. The instruction set from previous homework exercises is supplemented with additional instructions and used to code an assembly language program which has been used as a design example throughout the course. The resulting simulation provides a functional model of processor with data and control signal visibility at each clock cycle. 


\section{Single-bus Architecture}

Addressing mode and microprogrammed control exercises are based on a single-bus architecture from the course text, shown in Figure 1. Processor registers and memory are defined along with control signals and the datapath to create the resulting architecture is shown in Figure 2.

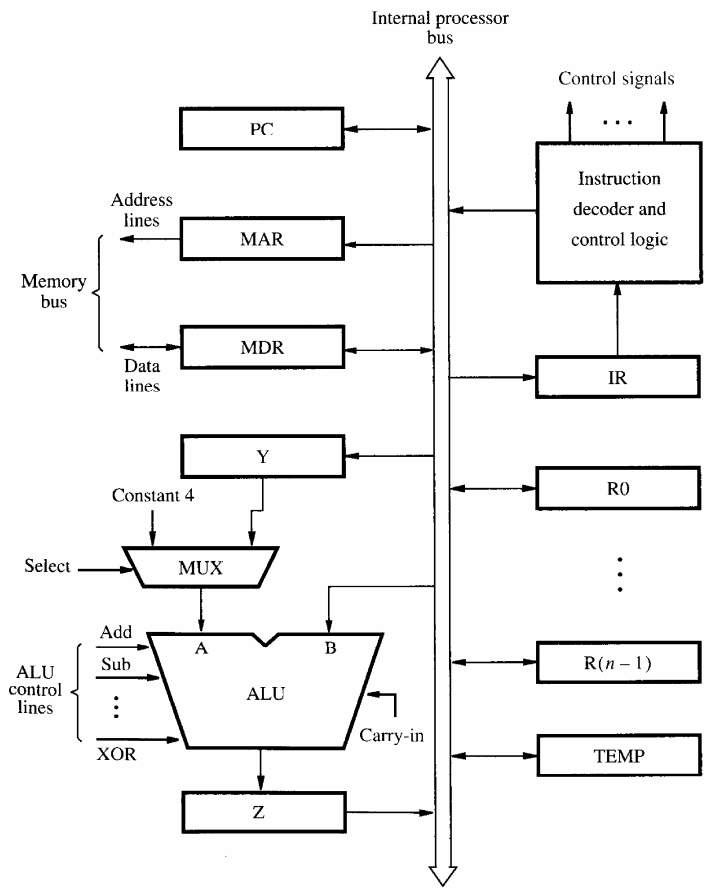

Figure 1. Single-bus architecture from text.

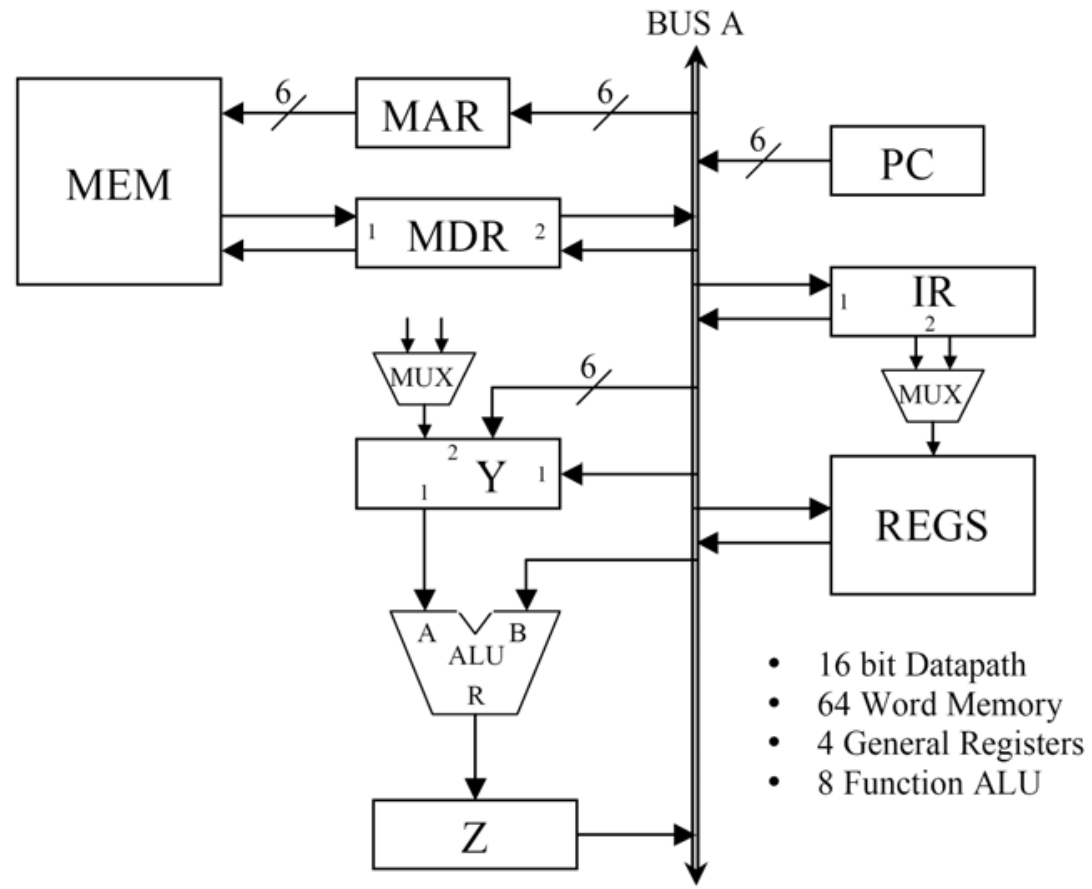

Figure 2. Single-bus architecture for homework. 


\section{Addressing Modes}

For the addressing mode homework exercise, a simple instruction format was created based on classroom and text examples. The instruction format, shown in Figure 3, is robust enough to demonstrate all the fundamental addressing modes and provides the basis for future exercises and the design project.

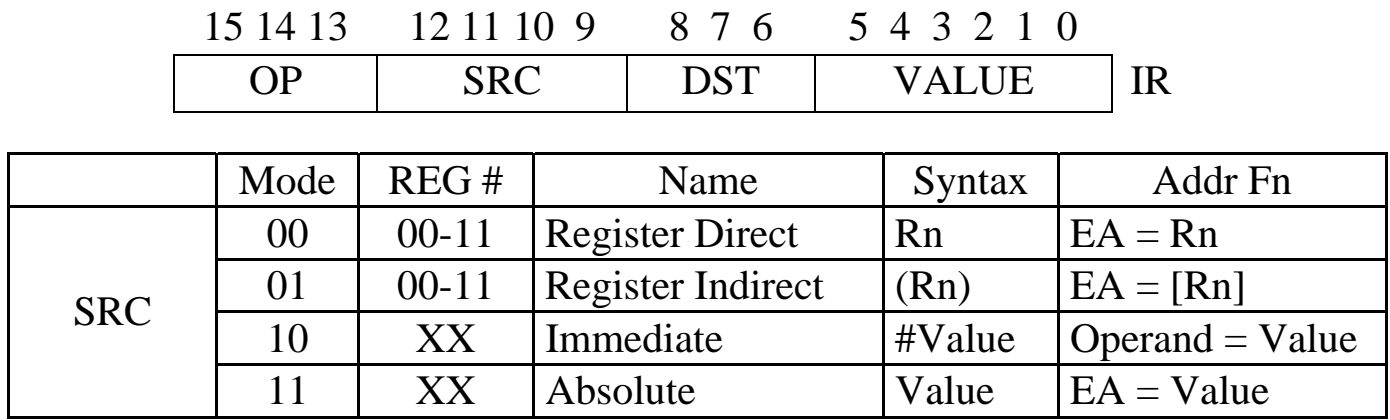

\begin{tabular}{|c|c|c|l|l|l|}
\hline & Mode & REG \# & \multicolumn{1}{c|}{ Name } & \multicolumn{1}{c|}{ Syntax } & \multicolumn{1}{c|}{ Addr Fn } \\
\hline \multirow{2}{*}{ DST } & 0 & $00-11$ & Register Direct & Rn & EA = Rn \\
\cline { 2 - 6 } & 1 & $\mathrm{XX}$ & Absolute & Value & EA = Value \\
\hline
\end{tabular}

\begin{tabular}{|c|c|l|l|}
\hline OP & Fn & Assembly Language & RTN \\
\hline 000 & MOVE & MOVE SRC,DST & DST $\leftarrow[$ SRC $]$ \\
\hline 111 & HALT & HALT & Halt $\leftarrow 1$ \\
\hline
\end{tabular}

Figure 3. Simple instruction format.

An assembly language program, shown in Figure 4, is used to test the various addressing modes and provides the students with hands-on application of the classroom concepts. The resulting contents of the registers and memory must be determined and then verified by simulation.

\begin{tabular}{|c|c|}
\hline 10VE & \#NUM2, RO \\
\hline MOVE & NUM1, R1 \\
\hline MOVE & $(\mathrm{RO}), \mathrm{R} 2$ \\
\hline MOVE & RO, NUM3 \\
\hline MOVE & $\#-1$, R3 \\
\hline & $(01)$ \\
\hline
\end{tabular}

Figure 4. Assembly language program.

\section{VHDL Components}

VHDL models of the various components of the architecture are provided to the students as a package. The students with basic knowledge of VHDL from their prerequisite digital logic course can use these models to create simulations of the architectural concepts. The most basic is the register, shown in Figure 5, which contains the data and control signals necessary to model the hardware component. 


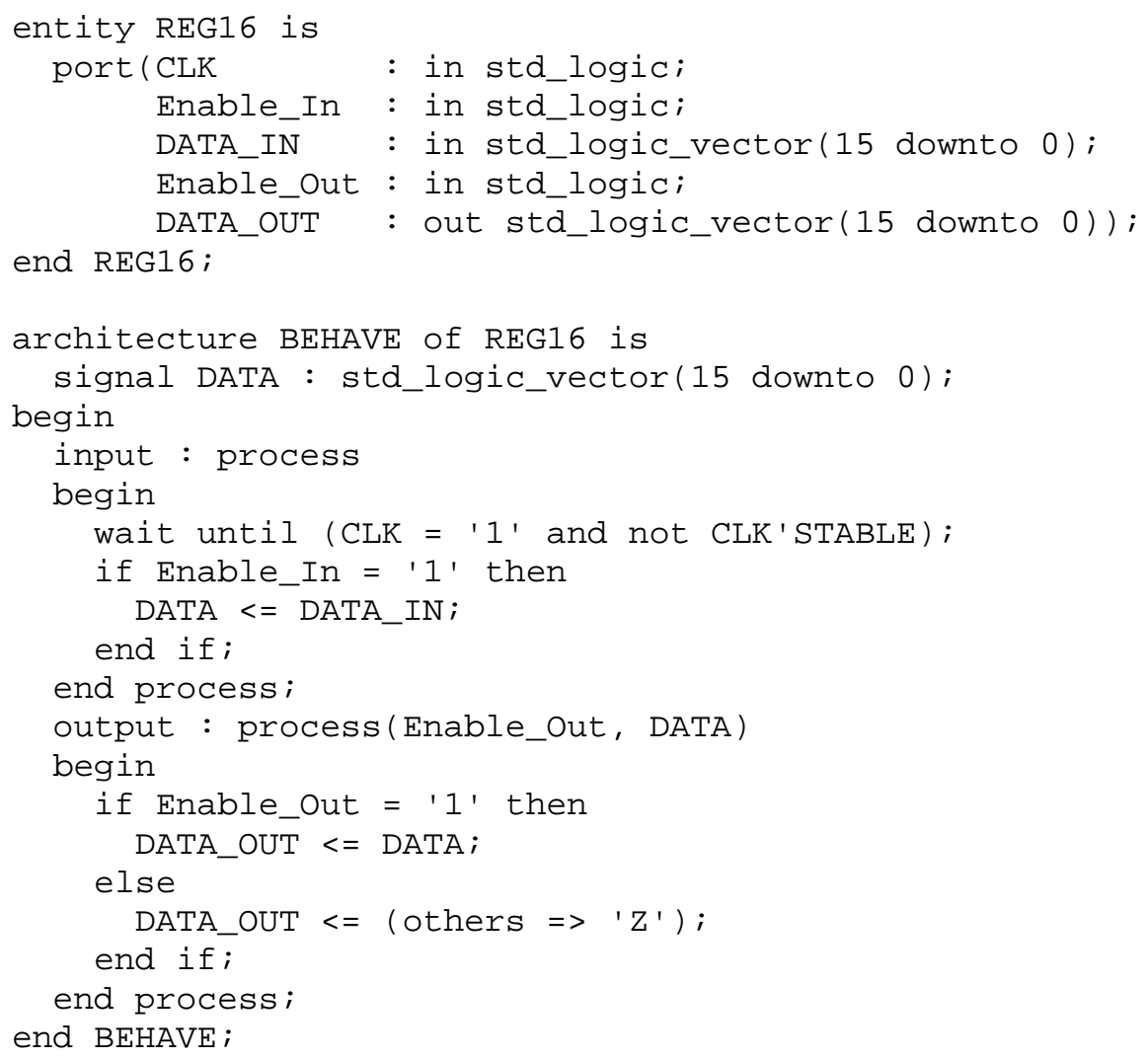

\section{Figure 5. VHDL model of a register.}

The key to successful simulation of various processor architectures and instruction sets is a memory model which can be programmed with machine code. This is accomplished using a VHDL generic which allows an initial contents to be set at time of instantiation. A memory dump feature has also been added to provide for easy verification of final memory contents after program execution. The VHDL memory model is shown in Figure 6 and a sample initialization is shown in Figure 7.

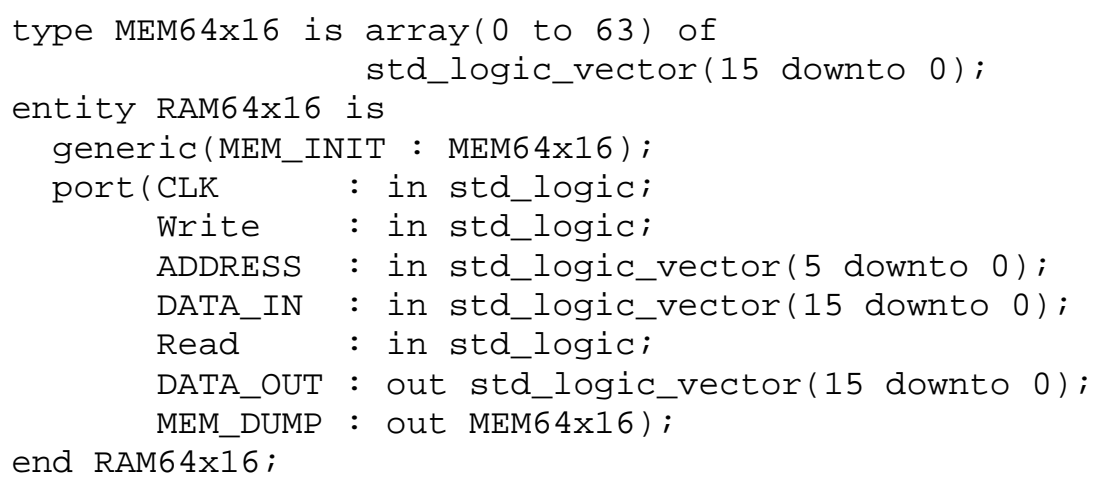

Figure 6. VHDL memory model. 


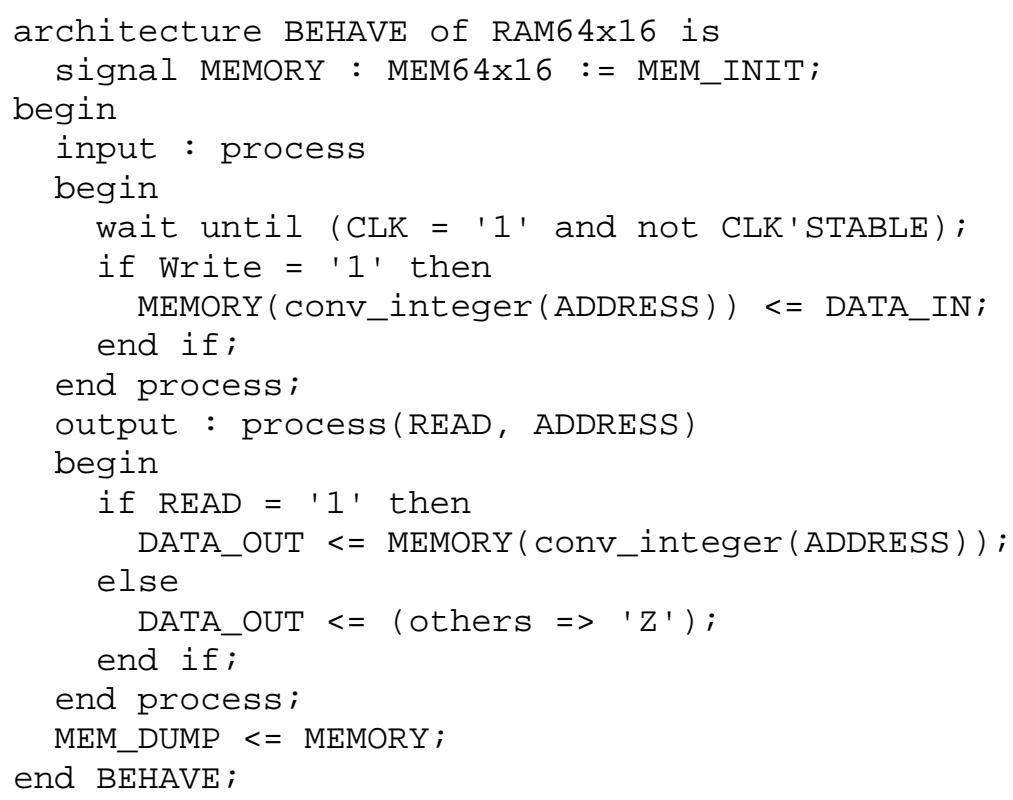

Figure 6 (cont). VHDL memory model.

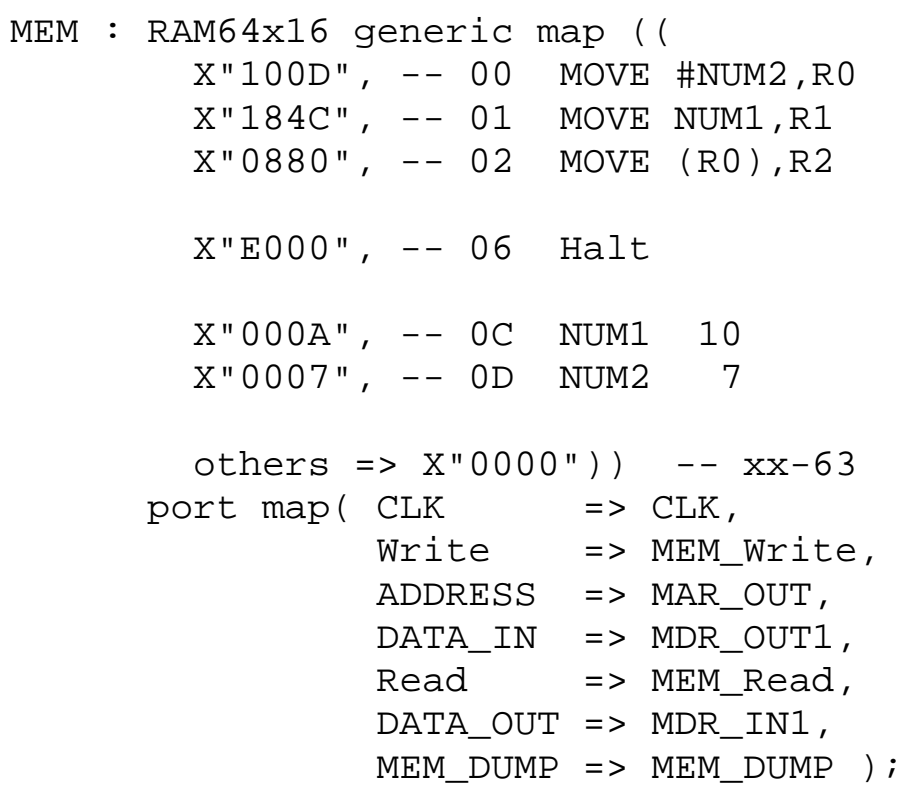

Figure 7. Memory instantiation and initialization.

\section{Control Signals}

The main VHDL is a structural model of the components to construct the architecture from Figure 2. Most of this VHDL is provided to the students, so they can concentrate on the control signals. The instruction set is implemented by modeling the control flow chart, shown in Figure 8, as a VHDL case statement, shown in Figure 9. 


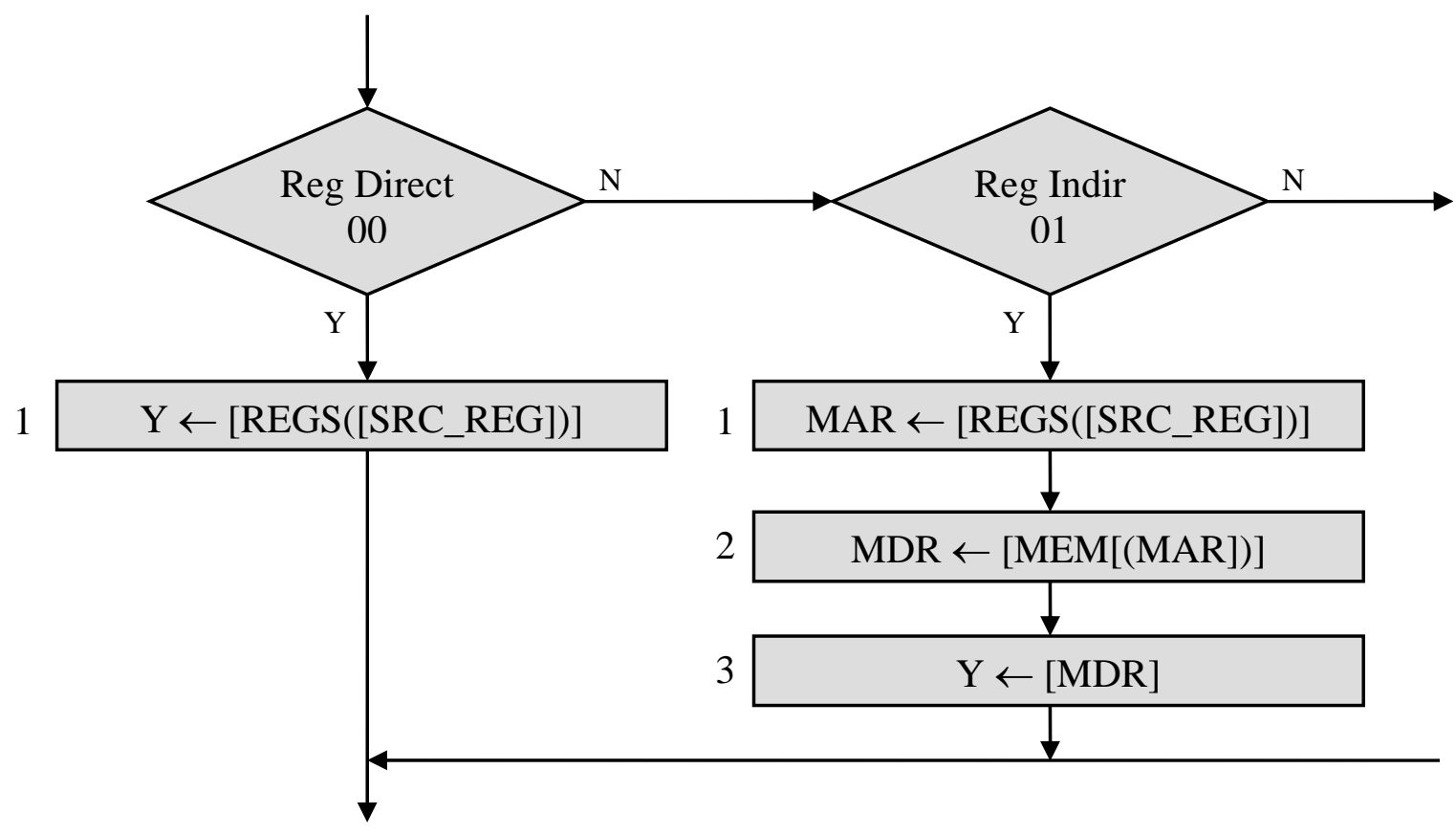

Figure 8. Control flow chart.

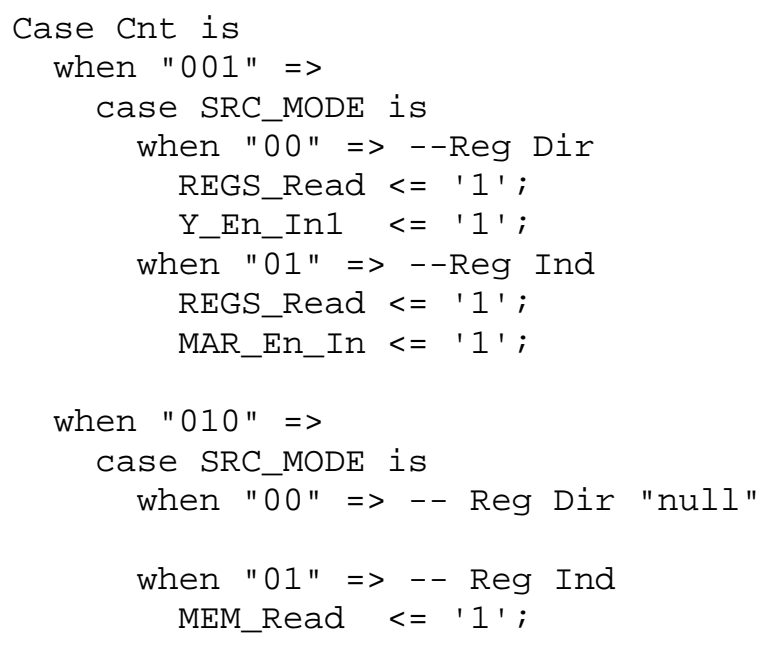

Figure 9. VHDL control model (abbreviated).

\section{VHDL Simulation}

Verification of the VHDL models is accomplished via simulation using an appropriate testbench. The testbench instantiates a copy of the main VHDL model and provides visibility of the key signals such as the control step count and instruction register. When execution is complete the final contents of the registers and memory are checked for the expected results. A portion of the testbench is shown in Figure 10 and an example simulation waveform is shown in Figure 11. 


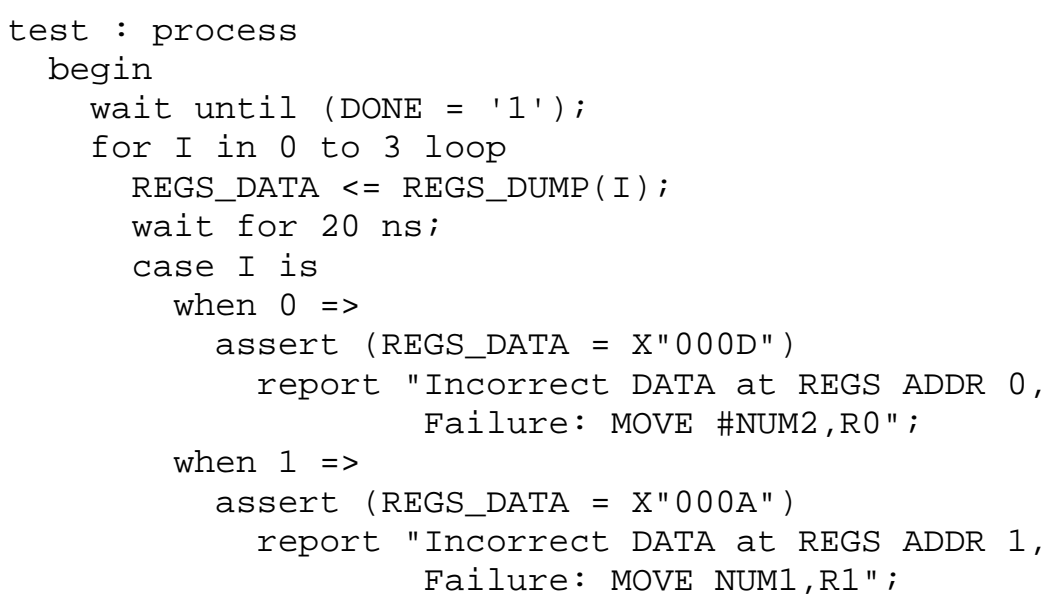

Figure 10. VHDL testbench.

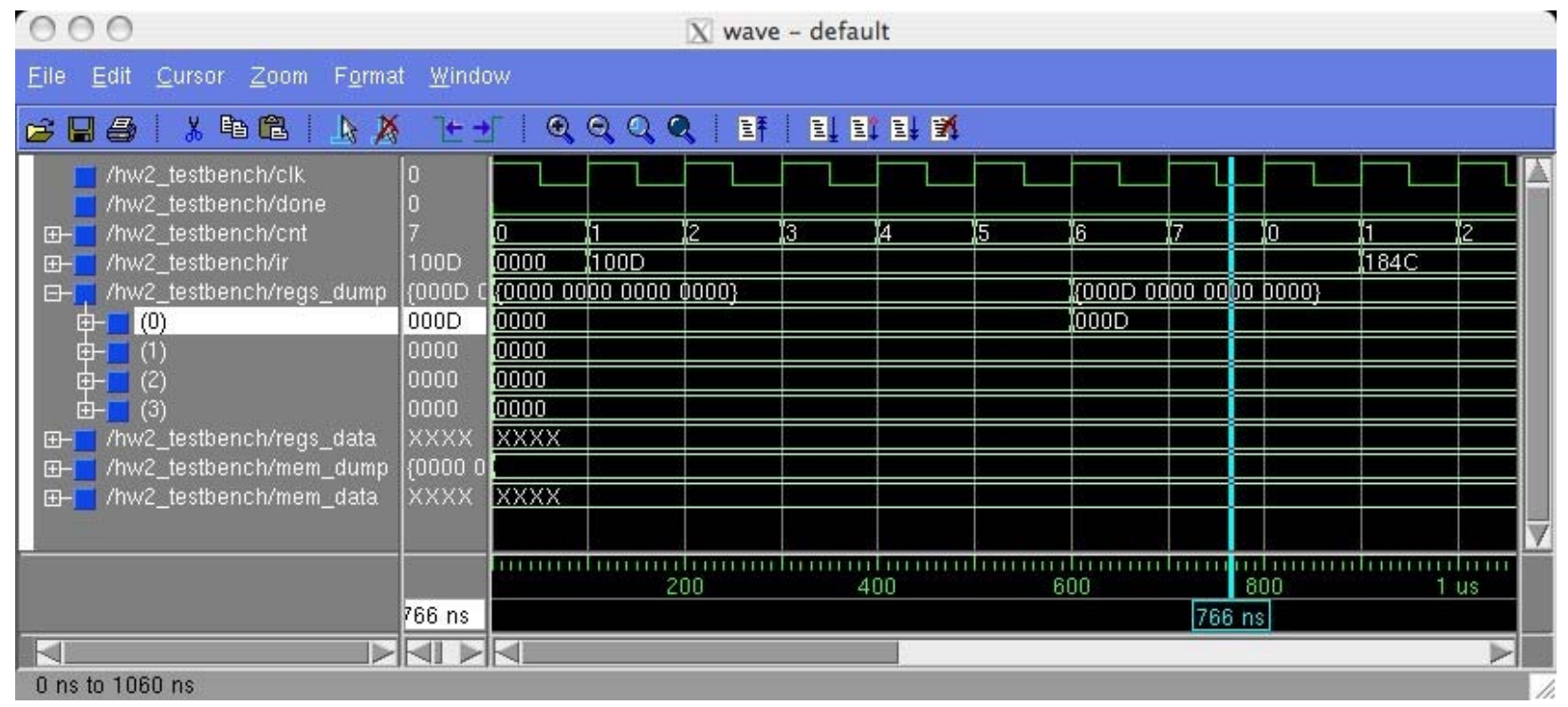

Figure 11. VHDL simulation waveform.

\section{Microprogrammed Control}

The next homework exercise builds on the previous one by adding the concept of microprogrammed control. The controller is again based on a text example, shown in Figure 12, which is readily adapted to the specific design, shown in Figure 13.

The single-bus architecture and instruction format remain the same, with the addition of the microinstruction format shown in Figure 14. The microinstruction format allows the students to write microcode to implement the control sequences. These microinstructions are loaded into the control store ROM of the microcontroller. 


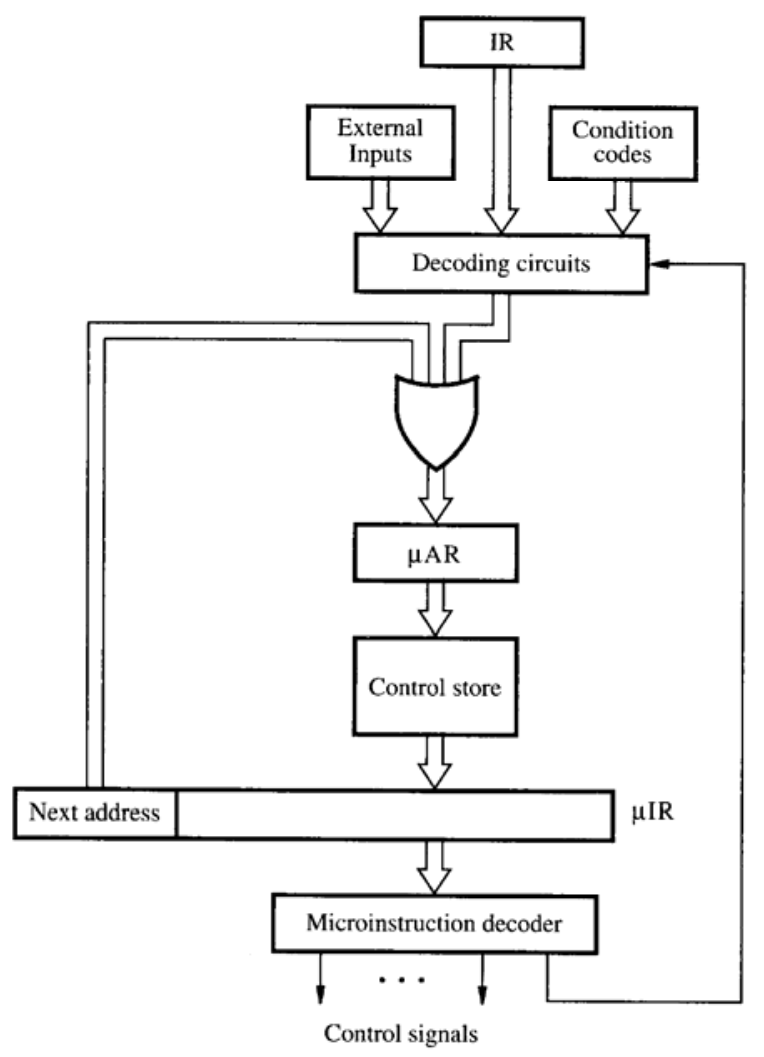

Figure 12. Microprogram controller from text.

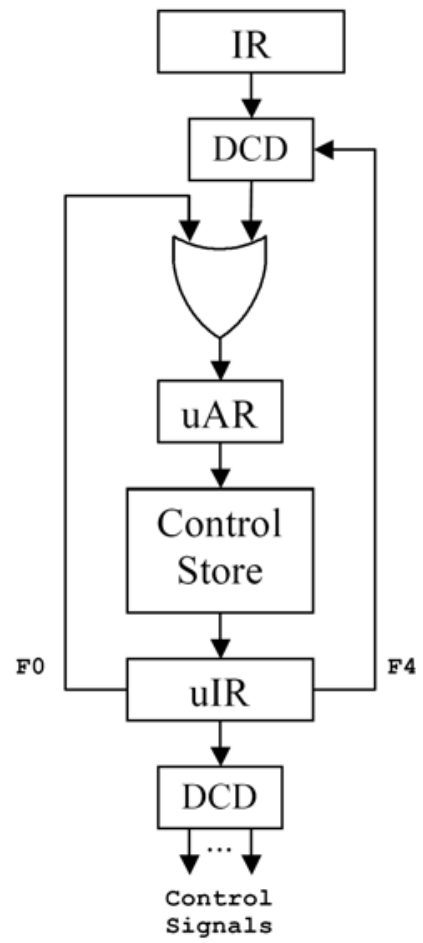

Figure 13. Microprogram controller for homework. 


\begin{tabular}{|c|c|c|c|c|}
\hline 151 & 987 & 654 & 32 & \\
\hline F0 & F1 & F2 & F3 & F4 \\
\hline
\end{tabular}

\begin{tabular}{|c|l|}
\hline \multicolumn{2}{|c|}{ F1 } \\
\hline $00 \odot$ & NOP \\
\hline 001 & PC_En_0ut \\
\hline 010 & MDR_En_0ut2 \\
\hline 011 & Z_En_In \\
\hline $10 \odot$ & MEM_Write \\
\hline 101 & MEM_Read \\
\hline 110 & REGS_Write \\
\hline 111 & REGS_Read \\
\hline
\end{tabular}

\begin{tabular}{|c|l|}
\hline \multicolumn{2}{|c|}{ F2 } \\
\hline $00 \odot$ & NOP \\
\hline 001 & IR_En_In1 \\
\hline 010 & MDR_En_In2 \\
\hline 011 & Y_En_In1 \\
\hline 100 & IR_En_0ut1 \\
\hline 101 & REGS_Sel \\
\hline 110 & PC_Inc \\
\hline 111 & Halt \\
\hline
\end{tabular}

\begin{tabular}{|l|l|}
\hline \multicolumn{2}{|c|}{ F3 } \\
\hline $0 \odot$ & NOP \\
\hline 01 & MAR_En_In \\
\hline 10 & Z_En_Out \\
\hline 11 & Y_En_In2 \\
\hline
\end{tabular}

\begin{tabular}{|c|}
\hline FO \\
\hline Addr of \\
next instr \\
\hline
\end{tabular}

\begin{tabular}{|c|c|c|}
\hline \multicolumn{2}{|r|}{$\mathrm{F} 4$} & OR Fn \\
\hline 00 & Next Addr & \\
\hline 01 & OR SRC & 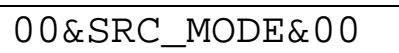 \\
\hline 10 & $\mathrm{OR}_{\mathrm{OP}}$ & ๑๑९\&OPCODE \\
\hline 11 & $0 R_{D S T}$ & OOO\&DST_MODE\&O९ \\
\hline
\end{tabular}

Figure 14. Microinstruction format.

\section{VHDL Model}

The existing VHDL models are supplemented with the addition of a ROM for the control store and decoders to produce the control signals. The control store is loaded with student generated microcode via the same generic construct used for the RAM. A sample of the ROM contents is shown in Figure 15.

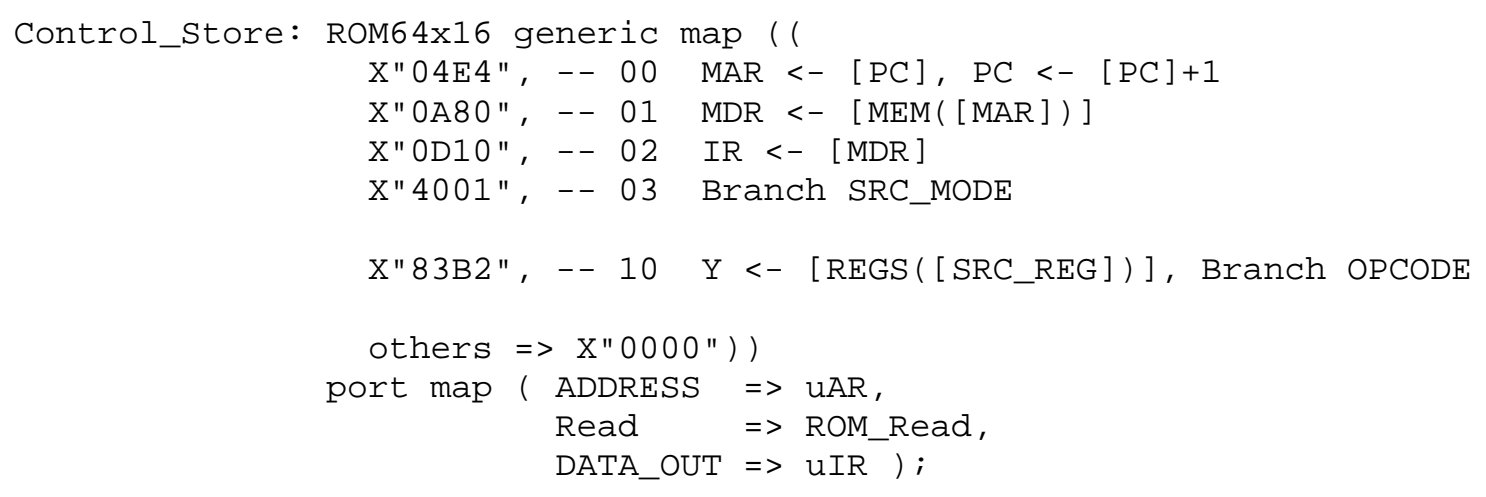

Figure 15. Control store ROM.

Due to the implementation of the control logic in the control store, the VHDL controller process is simplified to the reading of the microinstruction from ROM and the determination of the address of the next microinstruction, as shown in Figure 16. The testbench and simulation results follow directly from the addressing mode homework, using the same assembly language program and test data. 


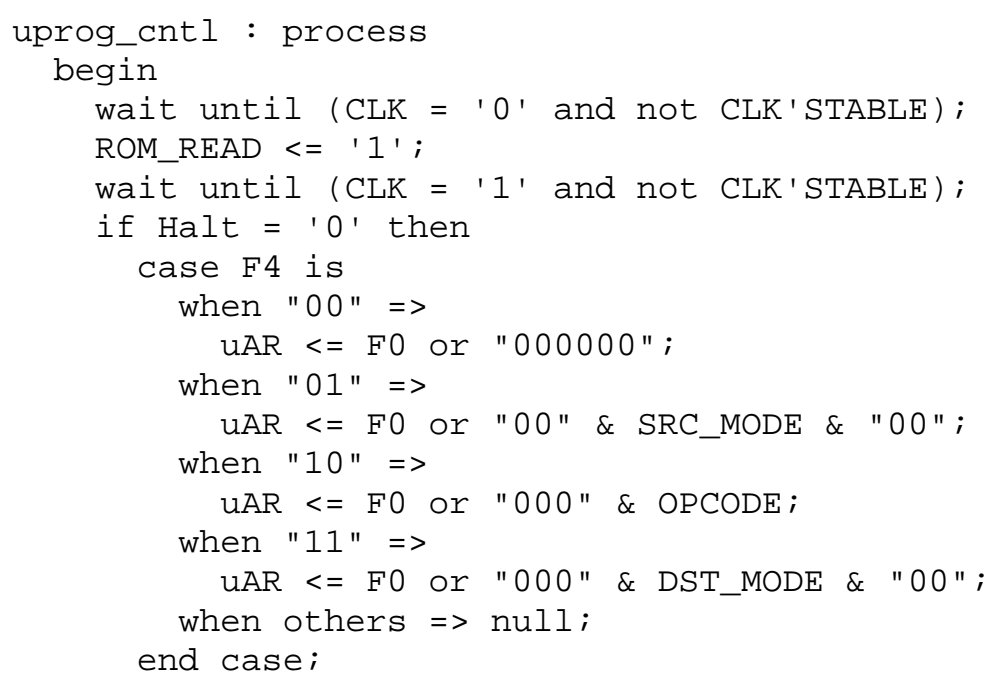

Figure 16. VHDL microprogram controller.

\section{Design Project}

The capstone design project ties together many of the concepts from throughout the course to produce a model of a simple processor capable of fetching and executing a sample assembly language program. The processor is based on a multiple-bus architecture example from the text, shown in Figure 17. Only minor modifications are necessary to create the design project architecture, shown in Figure 18.

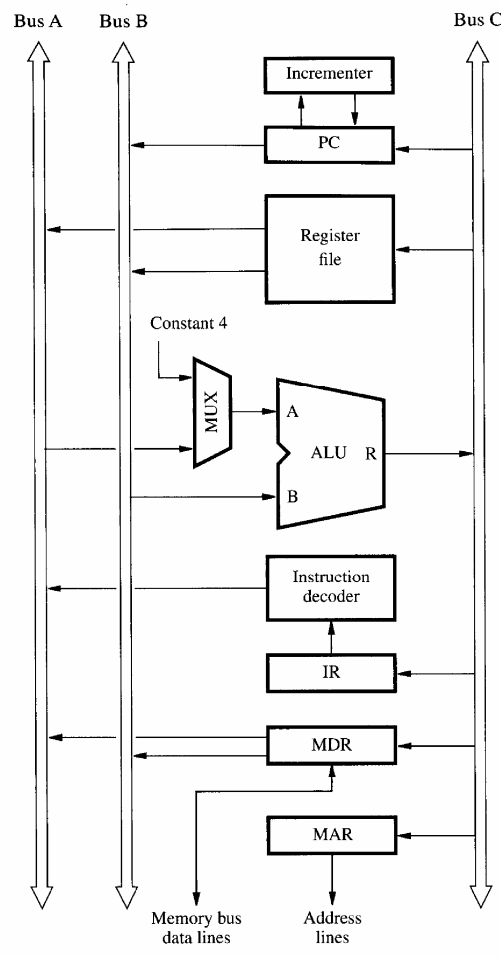

Figure 17. Multiple-bus architecture from text. 


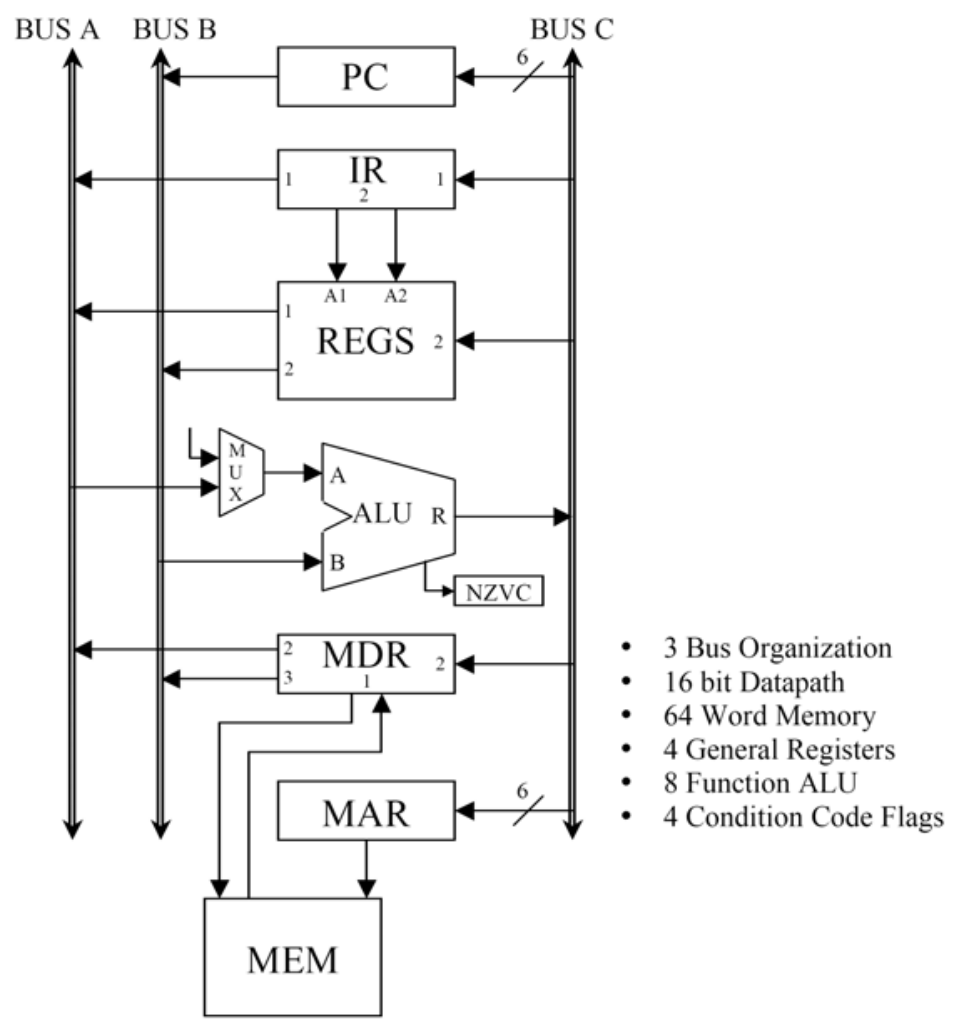

Figure 18. Design project architecture.

The design project computer uses a hardwired controller, again based on a text example shown in Figure 19. The VHDL model combines existing components to create the controller design shown in Figure 20.

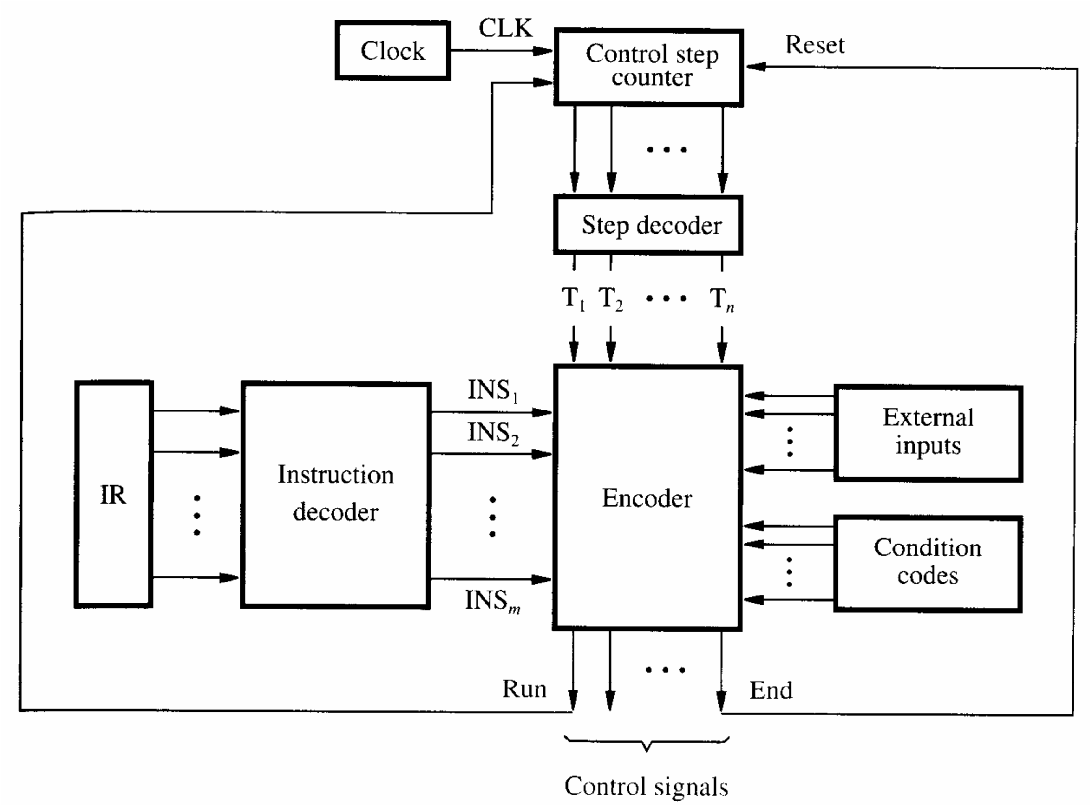

Figure 19. Hardwired controller from text. 


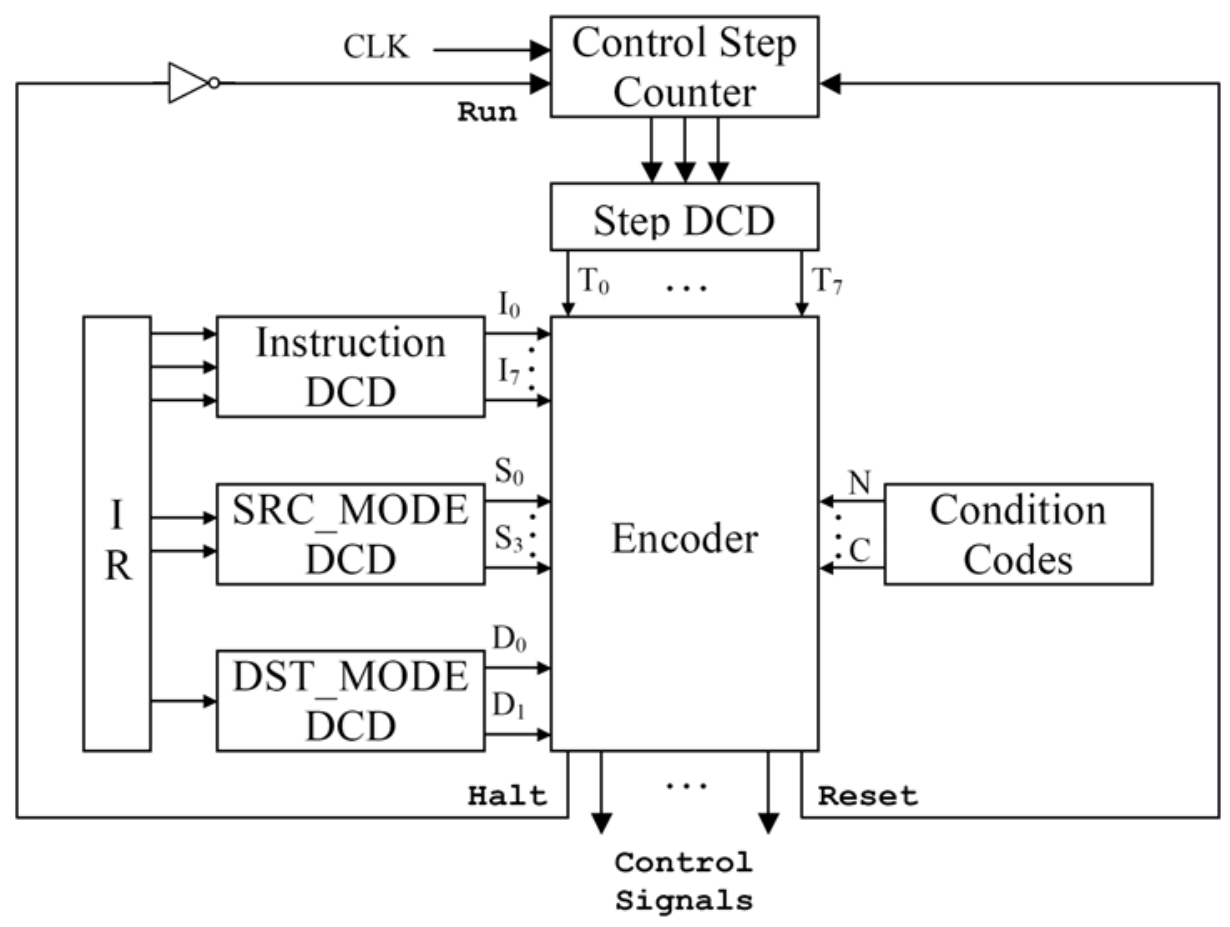

Figure 20. Controller for design project.

The instruction format and addressing modes remain the same as previous homeworks, with the addition of instructions for ADD and Branch. The expanded opcode table is shown in Figure 21. This expanded instruction set is used to create the assembly language program shown in Figure 22. The program uses multiple addressing modes and a loop to add a sequence of numbers. This basic program is used through the course as a recurring example to illustrate various concepts.

\begin{tabular}{|c|c|l|l|}
\hline OP & Fn & Assembly Language & RTN \\
\hline 000 & MOVE & MOVE SRC, DST & DST $<-[$ SRC] \\
\hline 001 & ADD & ADD SRC, DST & DST $<-[$ SRC] $+[$ DST] \\
\hline 110 & BGTZ & BGTZ DISP & PC $<-[$ PC] + DISP $($ CC $>0)$ \\
\hline 111 & HALT & HALT & Halt $<-1$ \\
\hline
\end{tabular}

Figure 21. Expanded opcodes for design project.

$$
\begin{aligned}
& \text { MOVE N, R1 } \\
& \text { MOVE \#NUM1, R2 } \\
& \text { MOVE } \# \Theta, R \odot \\
& \text { LOOP ADD (R2), RO } \\
& \text { ADD \#1, R2 } \\
& \text { ADD \#-1, R1 } \\
& \text { BGTZ LOOP } \\
& \text { MOVE R๑, SUM } \\
& \text { HALT }
\end{aligned}
$$

Figure 22. Assembly language program for design project. 


\section{VHDL Model}

The VHDL for the design project is again a structural model of the interconnection of the components from Figure 18. The main exercise for the students is the determination of the control signal logic using the method detailed in the text and class notes. The resulting VHDL controller structure is shown in Figure 23.

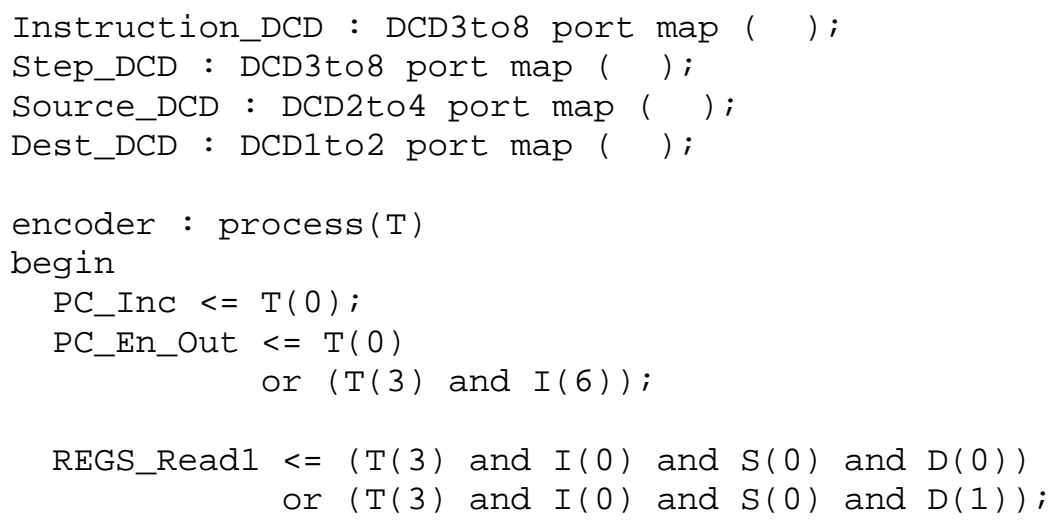

Figure 23. VHDL controller for design project.

VHDL testbench and simulation again follow directly from previous homeworks. Students can verify their controller design by examining simulation waveforms showing signal values and via register and memory dumps showing their final contents. A sample simulation waveform is shown in Figure 24.

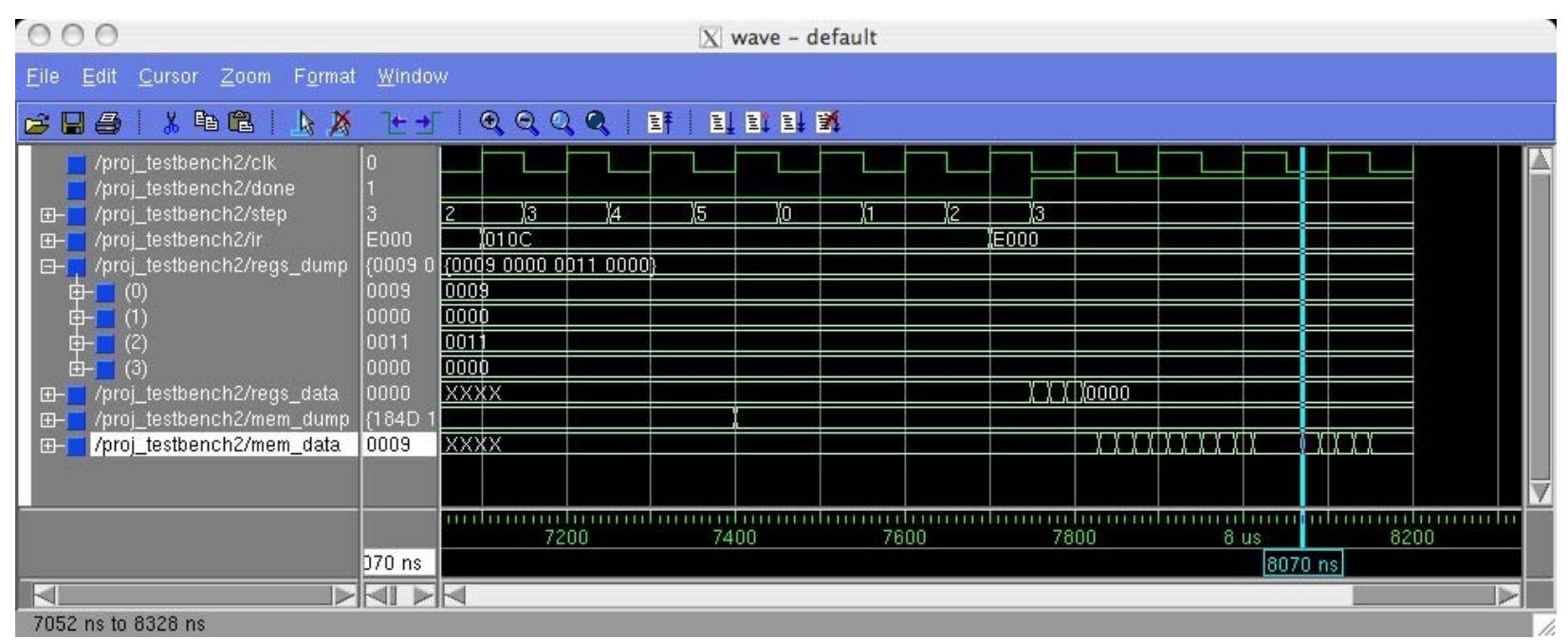

Figure 24. Design project simulation waveform.

\section{Results and Conclusions}

The VHDL homeworks and design project described in this paper have been successfully used to augment the classroom instruction for a pilot section of a computer architecture course. Student feedback has been very positive that the exercises provided direct reinforcement of classroom 
concepts and allowed them to visualize results via simulation. Approximately three-quarters of the students were able to successfully complete all homework assignments and over two-thirds were able to implement a completely functional design project.

The exercise design methodology was to combine/modify instructor provided VHDL models, rather than turning the course into a "programming" class. Student effort was focused on demonstration and understanding of classroom concepts by concentrating on the control signals and logic, rather than becoming engrossed in the VHDL. All architectural designs were derived directly from textbook examples providing reinforcement of concepts, as opposed to introduction of additional disparate material.

Again, the existing course text contains no coverage of VHDL. Hardware and architectural constructs were described in sufficient detail to allow easy modeling in VHDL by focusing on the "hardware description" characteristics of the language. The resulting combination of textbook and classroom instruction with VHDL modeling and simulation exercises provided students with a more robust learning experience and exposure to state-of-the-art design tools.

\section{Bibliography}

1. Hamacher, C., Z. Vranesic, and S. Zaky, Computer Organization, Fifth Edition, McGraw Hill, New York, NY, 2002. 